\title{
Combinatorial Preconditioners and Multilevel Solvers for Problems in Computer Vision and Image Processing*
}

\author{
Ioannis Koutis Gary L. Miller Ali Sinop David Tolliver \\ Computer Science Department \\ Carnegie Mellon University
}

November 6, 2009

\begin{abstract}
Linear systems and eigen-calculations on symmetric diagonally dominant matrices (SDDs) occur ubiquitously in computer vision, computer graphics, and machine learning. In the past decade a multitude of specialized solvers have been developed to tackle restricted instances of SDD systems for a diverse collection of problems including segmentation, gradient inpainting and total variation. In this paper we explain and apply the support theory of graphs, a set of of techniques developed by the computer science theory community, to construct SDD solvers with provable properties. To demonstrate the power of these techniques, we describe an efficient multigrid-like solver which is based on support theory principles. The solver tackles problems in fairly general and arbitrarily weighted topologies not supported by prior solvers. It achieves state of the art empirical results while providing robust guarantees on the speed of convergence. The method is evaluated on a variety of vision applications.
\end{abstract}

*This work was partially supported by the National Science Foundation under grant number CCF-0635257 and the University of Pittsburgh Medical Center under award number A-006461. 


\section{Introduction}

The Laplacian operator $\nabla^{2}$ has played a central role in computer vision for nearly 40 years. In Horn's early work, he employed finite element methods for elliptical operators in shape from shading [23], to produce albedo maps [24], and flow estimates [25]. In Witkin's seminal work [47] he studied the diffusion properties of matrix equations derived from $\nabla^{2}$ for linear filtering, later generalized by Perona and Malik [33] to the anisotropic case.

In recent years, combinatorial Laplacians of graphs have formed the algorithmic core of spectral methods [40, 32, 2, 48, 11, 18, 30, 12], random walks segmentation [18], in-painting [44, 31, 4], and matting methods [30]. Given the power of modern iterative solvers, we believe that reducing traditional image processing problems, such as Grady et al.'s work [20] on Mumford-Shah segmentation, to SDD systems at the inner loop is a critical endeavor. To this end, we note that non-linear filtering operations such as $\ell_{2}, \ell_{1}$ Total Variation $[37,10]$ and Non-Local Means [9, 8] can also be formulated as optimizations with these linear systems at their core. Further, we provide timing and modern complexity bounds for computer vision methods in $\S 5$ that require the solutions to SDD systems at their core.

From a practical standpoint, modern photos and videos, and medical images derived NMR and CT scanners provide enormously detailed portraits of a scene. As the resolution of imaging hardware has pushed at the limits of computational feasibility, researchers inevitably arrived at the study of iterative and hybrid solvers. Recently, vision and graphics researchers have developed specialized solvers [44, $16,4,31]$, and heuristic solvers with impressive empirical performance [39, 31, 21, 19]. In either case, the methods place strict requirements on the system, such as unit weight edges or 4-connectivity. For the methods that handle general weights, including Algebraic Multigrid (AMG) [38, 7], the solvers are based on heuristics and offer no guarantees on the speed of convergence. Indeed many applications, such as the spectral segmentation and convex programming, require wildly varying weights and often employ randomly sampled and loosely localized topologies. Furthermore, the heuristic nature of the solvers is generally undesirable in certain commercial applications, e.g. medical, where robust and timely behavior is a critical issue.

Given modern data volumes and reliability requirements it is clear that a SDD solver with provable convergence properties and sound theoretical machinery is important for the advancement and real-world success of methods based on linear system solutions. In this work we introduce the Combinatorial Multigrid Solver 
(CMG), a state of the art solver with provable properties. The CMG solver is based on principles of support theory for graphs, a set of techniques developed for the construction of combinatorial preconditioners, i.e. graphs that are simpler than a given graph and approximate it well in a precisely defined sense. An ancillary goal of this paper is to review certain useful fragments of support theory and apply them to analyze solvers.

\section{Support Theory for graphs}

Support Theory was developed for the study of Combinatorial subgraph preconditioners, introduced by Vaidya $[46,26]$. It has been at the heart of impressive theoretical results which culminated in the work of Spielman and Teng [43] who demonstrated that SDD systems can be solved in nearly-optimal $\tilde{O}\left(n \log { }^{O(1)} n\right)$ time and later in the work of Koutis and Miller [28] who formally proved that SDD matrices with planar connection topologies (e.g. 4-connectivity in the image plane) can be solved asymptotically optimally, in $O(n)$ time. We dub these solvers hybrid solvers since they combine algorithms and ideas from direct solvers, preconditioned Conjugate Gradient, and recursion.

\subsection{Reduction of SDDs to Laplacians}

A matrix $A$ is SDD if it is real symmetric and $A_{i i} \geq \sum_{j \neq i}\left|A_{i j}\right|$ for $1 \leq i \leq$ $n$. The Laplacian $A$ of a graph $G=(V, E, w)$, where $w$ is a non-negative weight function on the edges, is defined by $A_{i, j}=A(j, i)=-w_{i, j}$ and $A_{i, i}=$ $\sum_{i \neq j} w_{i, j}$. Thus Laplacians are SDD matrices having non-positive off diagonals and zero row sums. We briefly describe how any SDD system can be reduced to a Laplacian. SDD systems with positive off-diagonals can be reduced to the case of non-positive off diagonals using a very light-weight reduction known as the double-cover construction [22]. Assuming now negative off-diagonals, nodes with positive row sums can be viewed as nodes that have an implicit edge to a new "grounded node". In general they do not cause any significant changes in the Laplacian solver [5, 27].

\subsection{Preconditioners - Motivating Support Theory}

Iterative algorithms, such as the Chebyshev iteration or the Conjugate Gradient, converge to a solution using only matrix-vector products with $A$. It is well known 
that iterative algorithms suffer from slow convergence properties when the conditioning of $A, \kappa(A)$, - defined as the ratio of the largest over the minimum eigenvalue of $A$ - is large [1].

Preconditioned iterative methods attempt to remedy the problem by changing the linear system to $B^{-1} A x=B^{-1} b$. In this case, the algorithms use matrixvector products with $A$, and solve linear systems of the form $B y=z$. The speed of convergence now depends on the condition number $\kappa(A, B)$, defined as

$$
\kappa(A, B)=\max _{x} \frac{x^{T} A x}{x^{T} B x} \cdot \max _{x} \frac{x^{T} B x}{x^{T} A x}
$$

where $x$ is taken to be outside the null space of $A$. In constructing a preconditioner $B$, one has to deal with two contradictory goals: (i) Linear systems in $B$ must be easier than those in $A$ to solve, (ii) The condition number must be small to minimize the number of iterations.

Historically, preconditioners were natural parts of the matrix $A$. For example, if $B$ is taken as the diagonal of $A$ we get the Jacobi Iteration, and when $B$ is the upper triangular part of $A$, we get the Gauss-Seidel iteration.

The cornerstone of combinatorial preconditioners is the following intuitive yet paradigm-shifting idea explicitly proposed by Vaidya: A preconditioner for the Laplacian of a graph $A$ should be the Laplacian of a simpler graph $B$, derived in a principled fashion from $A$.

\subsection{Graphs as electric networks - Support basics}

There is a fairly well known analogy between graph Laplacians and resistive networks [13]. If $G$ is seen as an electrical network with the resistance between nodes $i$ and $j$ being $1 / w_{i, j}$, then in the equation $A v=i$, if $v$ is the vector of voltages at the node, $i$ is the vector of currents. Also, the quadratic form $v^{T} A v=\sum_{i, j} w_{i, j}\left(v_{i}-v_{j}\right)^{2}$ expresses the power dissipation on $G$, given the node voltages $v$. In view of this, the construction of a good preconditioner $B$ amounts to the construction of a simpler resistive network (for example by deleting some resistances) with an energy profile close to that of $A$.

The support of $A$ by $B$, defined as $\sigma(A / B)=\max _{v} v^{T} A v / v^{T} B v$ is the number of copies of $B$ that are needed to support the power dissipation in $A$, for all settings of voltages. The principal reason behind the introduction of the notion of support, is to express its local nature, captured by the Splitting Lemma.

Lemma 2.1. [Splitting Lemma] If $A=\sum_{i=1}^{m} A_{i}$ and $B=\sum_{i=1}^{m} B_{i}$, where $A_{i}, B_{i}$ are Laplacians, then $\sigma(A, B) \leq \max _{i} \sigma\left(A_{i}, B_{i}\right)$. 
The Splitting Lemma allows us to bound the support of $A$ by $B$, by splitting the power dissipation in $A$ into small local pieces, and "supporting" them by also local pieces in $B$.

For example, in his work Vaidya proposed to take $B$ as the maximal weight spanning tree of $A$. Then, it is easy to show that $\sigma(B, A) \leq 1$, intuitively because more resistances always dissipate more power. In order to bound $\sigma(A, B)$, the basic idea to let the $A_{i}$ be edges on $A$ (the ones not existing in $B$ ), and let $B_{i}$ be the unique path in the tree that connects the two end-points of $A_{i}$. Then one can bound separately each $\sigma\left(A_{i}, B_{i}\right)$. In fact, it can be shown that any edge in $A$ that doesn't exist in $B$, can be supported only by the path $B_{i}$.

As a toy example, consider the example in Figure 1(a) of the two (dashed) edges $A_{1}, A_{2}$ and their two paths in the spanning tree (solid) that share one edge $e$.

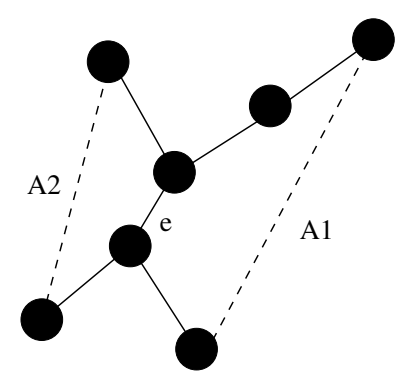

(a) A graph and its spanning tree - obtained by deleting the dashed edges.

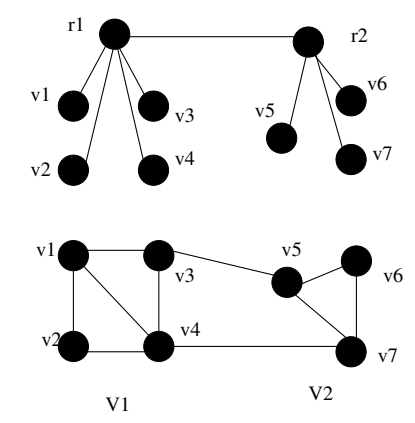

(b) A graph and its Steiner preconditioner

Figure 1:

In this example, the dilation of the mapping is equal to 3 , i.e. the length of the longest of two paths. Also, as $e$ is uses two times, we say that the congestion of the mapping is equal to 2. A core Lemma in Support Theory [3, 5] is that the support can be upper bounded by the product congestion $*$ dilation.

\subsection{Steiner preconditioners}

Steiner preconditioners, introduced in [22] and extended in [29] introduce external nodes into preconditioners. The proposed preconditioner is based on a partitioning of the $n$ vertices in $V$ into $m$ vertex-disjoint clusters $V_{i}$. For each $V_{i}$, the 
preconditioner contains a star graph $S_{i}$ with leaves corresponding to the vertices in $V_{i}$ rooted at a vertex $r_{i}$. The roots $r_{i}$ are connected and form the quotient graph $Q$. This general setting is illustrated in Figure 1(b), consisting of good clusters.

Let $D^{\prime}$ be the total degree of the leaves in the Steiner preconditioner $S$. Let the restriction $R$ be an $n \times m$ matrix, where $R(i, j)=1$ if vertex $i$ is in cluster $j$ and 0 otherwise. Then, the Laplacian of $S$ has $n+m$ vertices, and the algebraic form

$$
S=\left(\begin{array}{cc}
D^{\prime} & -D^{\prime} R \\
-R^{T} D^{\prime} & Q+R^{T} D^{\prime} R
\end{array}\right)
$$

A worrying feature of the Steiner preconditioner $S$ is the extra number of vertices. So how do we even use it? Gremban and Miller [22] proposed that every time a system of the form $B z=y$ is solved in an usual preconditioned method, the system $S\left(\begin{array}{c}z \\ z^{\prime}\end{array}\right)=\left(\begin{array}{c}y \\ 0\end{array}\right)$ should be solved instead, for a set of don't care variables $z^{\prime}$. They also showed that the operation is equivalent to preconditioning with the dense matrix

$$
B=D^{\prime}-V\left(Q+D_{Q}\right)^{-1} V^{T}
$$

where $V=D^{\prime} R$, and $D_{Q}=R^{T} D^{\prime} R$. The matrix $B$ is called the Schur complement of $S$ with respect to the elimination of the roots $r_{i}$, further it is a well known that $B$ is also a Laplacian.

The analysis of the support $\sigma(A / S)$, is identical to that for the case of subgraph preconditioners. For example, going back to Figure $1(\mathrm{~b})$, the edge $\left(v_{1}, v_{4}\right)$ can only be supported by the path $\left(v_{1}, r_{1}, v_{4}\right)$, and the edge $\left(v_{4}, v_{7}\right)$ only by the path $\left(v_{4}, r_{1}, r_{2}, v_{7}\right)$. Similarly we can see the mappings from edges in $A$ to paths in $S$ for every edge in $A$. In the example, the dilation of the mapping is 3 , and it can be seen that to minimize the congestion on every edge of $S$ (i.e. make it equal to 1 ), we need to take $D^{\prime}=D$, where $D$ are the total degrees of the nodes in $A$, and $w\left(r_{1}, r_{2}\right)=w\left(v_{3}, v_{5}\right)+w\left(v_{4}, v_{7}\right)$. More generally, for two roots $r_{i}, r_{j}$ we should have $w\left(r_{i}, r_{j}\right)=\sum_{i^{\prime} \in V_{i}, j^{\prime} \in V_{j}} w_{i, j}$. Under this construction, the algebraic form of the quotient $Q$ can be seen to be $Q=R^{T} A R$.

So far no special properties of the clustering have been used. Those come into play in bounding the support of $S$ by $A, \sigma(S / A)$. In [29] it was shown that the support $\sigma(S / A)$ reduces to bounding the support $\sigma\left(S_{i}, A\left[V_{i}\right]\right)$, for all $i$, where $A\left[V_{i}\right]$ denotes the graph induced in $A$ by the vertices $V_{i}$. When are these bounded? Before we answer this question, let us recall the definition of conductance. 
Definition 2.2. The conductance $\phi(A)$ of a graph $A=(V, E, w)$ is defined as

$$
\phi(A)=\min _{S \subseteq V} \frac{w(S, V-S)}{\min (w(S), w(V-S))}
$$

where $w(S, V-S)$ denotes the total weight connecting the sets $S$ and $V-S$, and where $w(S)$ denotes the total weight incident to the vertices in $S$.

The main result of [29] is captured by the following Theorem.

Theorem 2.3. The support $\sigma(S / A)$ is bounded by a constant $c$ independent from $n$, if and only for all $i$ the conductance of the graph $A^{o}\left[V_{i}\right]$ induced by the nodes in $V_{i}$ augmented by the edges leaving $V_{i}$ is bounded by a constant $c^{\prime}$.

Although Theorem 2.3 doesn't give a way to pick to clusters, it does provide a way to avoid bad clusterings.

\subsection{Support Theory \& Grady's clusterings}

In recent work [19], Grady proposed a multigrid method where the construction of the "coarse" grid follows exactly the construction of the quotient graph in the previous section. Specifically, Grady proposes a clustering such that every cluster contains exactly one of certain pre-specified "coarse" nodes. He then defines the restriction matrix $R$ and he lets the coarse grid be $Q=R^{T} A R$, identically to the construction of the previous Section. The question then is whether the proposed clustering provides the guarantees that by Theorem 2.3 are necessary to construct a good Steiner preconditioner. In the following Figure, we replicate Figure 2 of [19], with a choice of weights that force the depicted clustering.

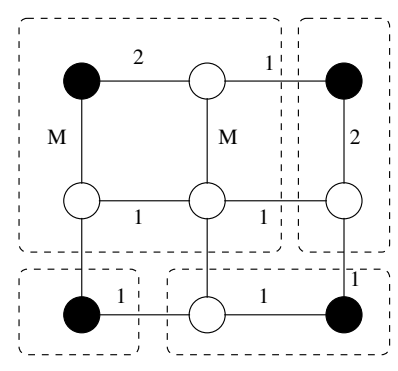

Figure 2: A bad clustering 
Every cluster in Figure 2 contains exactly one black/coarse node. The problem with the clustering is that the top left cluster, has a very low conductance when $M>>1$. In general, in order to satisfy the requirement of Theorem 2.3, there are cases where the clustering has to contain clusters with no coarse nodes in them.

It is interesting that the Maximally Connected Neighbor (MCN) algorithm proposed in [19] comes very close to the clustering algorithm proposed in [29]. Of course, it is imaginable that there are instances where MCN may not induce bad clusterings. On such instances, Grady's clustering has provable properties. Grady's solver is a multigrid solver, but as we will see in Section 3, multigrid solvers and Steiner preconditioners are closely related.

\section{The Combinatorial Multigrid Solver}

In this section we describe the Combinatorial Multigrid Solver. As we will see, the CMG solver matches the simple form of AMG, but with two distinguishing features: (i) The "coarsening" strategy is markedly different; it is in fact easier to implement and faster than the various AMG coarsening strategies. (ii) The algorithm is truly "black-box", in stark contrast which AMG which employs an extensive list of algorithmic knobs.

\subsection{A graph decomposition algorithm}

According to the discussion of $\S 2.4$, the crucial step for the construction of a good Steiner preconditioner is the computation of a group decomposition that satisfies, as best as possible, the requirements of Theorem 2.3. Before the presentation of the Decompose-Graph algorithm, that extends the ideas of [29], we need to introduce a couple of definitions. Let $\operatorname{vol}_{G}(v)$ denote the total weight incident to node $v$ in graph $G$. The weighted degree of a vertex $v$ is defined as the ratio

$$
w d(v)=\frac{\operatorname{vol}(v)}{\max _{u \in N(v)} w(u, v)} .
$$

The average weighted degree of the graph is defined as awd $(G)=(1 / n) \sum_{v \in V} w d(v)$.

It is not very difficult to prove that the algorithm Decompose-Graph produces a partitioning where the conductance of each cluster depends only on $\operatorname{awd}(A)$ and the constant $\kappa$. In fairly general topologies that allow high degree nodes, $\operatorname{awd}(A)$ is constant and the number of clusters $m$ returned by the algorithm is such that $n / m>2$ (and in practice larger than 3 or 4 ). There are many easy ways to 


\section{Algorithm Decompose-Graph}

Input: Graph $A=(V, E, w)$

Output: Disjoint Clusters $V_{i}$ with $V=\bigcup_{i} V_{i}$

1. Let $W \subseteq V$ be the set of nodes satisfying $w d(v)>\kappa \cdot \operatorname{awd}(A)$, for some constant $\kappa>4$.

2. Form a forest graph $F$, by keeping the heaviest incident edge of $v$ for each vertex $v \in V$ in $A$.

3. For every vertex $w \in W$ such that $\operatorname{vol}_{T}(w)<\operatorname{vol}_{G}(w) / \operatorname{awd}(A)$ remove from $F$ the edge contributed by $w$ in Step 2 .

4. Decompose each tree $T$ in $F$ into vertex-disjoint trees of constant conductance.

implement Step 3. Our current implementation makes about three passes of $A$. Of course, one can imagine variations of the algorithm (i.e. a correction step, etc) that may make the clustering phase a little more expensive with the goal of getting a better conductance and an improved condition number, if the application at hand requires many iterations of the solver.

\subsection{From Steiner preconditioners to Multigrid}

Multigrid algorithms have been a very active research area for nearly three decades. There are many expository article and books, among which [45]. In order to describe the reasoning that leads to our Combinatorial Multigrid Algorithm, we will need to shortly review the basic principles behind the generic two-level iteration.

Algebraically, any of the classic preconditioned iterative methods, such as the Jacobi and Gauss-Seidel iteration, is nothing but a matrix $\mathcal{S}$, which gets applied implicitly to the current error vector $e$, to produce a new error vector $e^{\prime}=\mathcal{S} e$. For example, in the Jacobi iteration we have $\mathcal{S}=\left(I-D^{-1} A\right)$. This has the effect that it reduces effectively only part of the error in a given iterate, namely the components that lie in the low eigenspaces of $\mathcal{S}$ (usually referred to as high frequencies of $A$ ). The main idea behind a two-level multigrid is that the current smooth residual error $r=b-A x$, can be used to calculate a correction $P^{T} Q^{-1} P r$, where $Q$ is a smaller graph and $P$ is an $m \times n$ restriction operator. The correction is then added to the iterate $x$. The hope here is that for smooth residuals, the low-rank matrix $P^{T} Q^{-1} P$ is a good approximation of $A^{-1}$. Algebraically, this correction is the application of the operator $T=\left(I-P^{T} Q^{-1} P A\right)$ to the error 
vector $e$. The choice of $P$ and $Q$ is such that $T$ is a projection operator with respect to the $A$-inner product, a construction known as the Galerkin condition. Two-level convergece proofs are then based on bounds on the angle between the subspace $N u l l(P)$ and the high frequency subspace of $\mathcal{S}$.

At a high level, the key idea behind CMG is that the provably small condition number $\kappa(A, B)$ where $B$ is given in expression 3 , is equal to the condition number $\kappa(\hat{A}, \hat{B})$ where $\hat{A}=D^{-1 / 2} A D^{-1 / 2}$ and $\hat{B}=D^{-1 / 2} B D^{-1 / 2}$. This in turn implies a bound on the angle between the low frequency of $\hat{A}$ and the high frequency of $\hat{B}$ [29]. The latter subspace includes $\operatorname{Null}\left(R^{T} D^{1 / 2}\right)$. This fact suggests to choose $R^{T} D^{1 / 2}$ as the projection operator while performing relaxation with $(I-\hat{A})$ on the system $\hat{A} y=D^{-1 / 2} b$, with $y=D^{1 / 2} x$. Combining everything, we get the following two-level algorithm.

\section{Two-level Combinatorial Multigrid}

Input: Laplacian $A=(V, E, w)$, vector $b$, approximate solution $x, n \times m$ restriction matrix $R$

Output: Updated solution $x$ for $A x=b$

1. $D:=\operatorname{diag}(A) ; \hat{A}:=D^{-1 / 2} A D^{-1 / 2}$;

2. $z:=(I-\hat{A}) D^{1 / 2} x+D^{-1 / 2} b$;

3. $r:=D^{-1 / 2} b-\hat{A} z ; w:=R^{T} D^{1 / 2} r$;

4. $Q:=R^{T} A R ;$ Solve $Q y=w$;

5. $z:=z+D^{1 / 2} R y$

5. $x:=D^{-1 / 2}\left((I-\hat{A}) z+D^{-1 / 2} b\right) z$

The two-level algorithm can naturally be extended into a full multigrid algorithm, by recursively calling the algorithm when the solution to the system with $Q$ is requested.

\section{SDDs arising in Computer Vision}

Many computer vision problems naturally suggest a graph structure - for example the vertices often correspond to samples (e.g. pixels, patches, images), the edge set establishes pairwise comparisons or constraints encoded in the graph and the weights are either data driven (for clustering) or the result of an ongoing optimization procedure (weights in the $t^{\text {th }}$ iteration of Newton's method).

The reformulation of fundamental objective functions in computer vision, such as the recent reduction of the Mumford-Shah functional in [20], to optimizations 
on combinatorial graphs opens the door to faster and more accurate algorithms. In this section we illustrate the pervasiveness and utility of SDDs with a collection of related problems that reduce to solutions to SDDs in the inner loop. For the two Total Variation applications we demonstrate that the systems reduce to SDDs at their core - for the others this fact is obvious.

\subsection{Gradient Inpainting}

Recent work on gradient inpainting [44, 31, 4] has centered around the development of specialized solvers for 4-connected meshes (either weighted or unweighted). The gradient inpainting problem seeks to integrate out an image from a (potentially sparse) set of image gradients. Formulated as a least squares optimization, given a vector of gradient constraints $\Delta^{c}$ for each channel $c$, the corresponding set of relationships encoded in the edge-node incidence matrix $\Gamma$ and a possible weighting of the constraints $W$ - the least squares image is obtained by solving:

$$
\Gamma^{T} W \Gamma x^{c}=\Gamma^{T} W^{-1 / 2} \Delta^{c}
$$

for each color channel. As $\Gamma^{T} W \Gamma$ is a weighted Laplacian the solution can be computed in $\tilde{O}(n)$ work for the general case and $O(n)$ work the weighted planar case (as addressed in [44, 31, 4]) - the image reconstruction step requires less work than linear filtering.

The gradient inpainting problem is evocative of Total Variation (TV) approaches for image processing problems. In the next section we outline optimization of related denoising functionals which can be used to condition least squares inpainting.

\subsection{Non-linear Filtering and Convex Optimization}

Non-linear methods in image processing based on Total Variation ${ }^{1}$ (TV) arise in image denoising [37], super resolution [35], and inpainting [34] in computer vision. In this section we demonstrate that $\ell_{2}, \ell_{2}$ and anisotropic (i.e. Manhattan) $\ell_{2}, \ell_{1}$ TV-like functionals reduce to solving SDD systems and can thus be integrated into $\mathrm{CV}$ pipelines at a cost comparable to applying a non-separable filterbank.

\footnotetext{
${ }^{1}$ See [10] for a thorough survey of TV and related mathematical image processing methods.
} 
We begin with the discrete (anisotropic) form of the Rudin, Osher, Fatemi TV functional [37], for $p$-norms along the $\ell_{1}$ to $\ell_{2}$ continuum:

$$
\min _{x}:\|x-s\|_{2}^{2}+\lambda\|\nabla x\|_{p}
$$

where $\ell_{p} \mid 2 \geq p \geq 1$ maintains the structural property of convexity, the existence of efficient algorithms, and generalizes to weighted norms. We outline a method for the $p=1$ case as work on the statistics of natural images [36] and the success of sparse representations motivate the use of $\ell_{1}$ for penalizing the image gradients. Write the primal for $\ell_{2}, \ell_{1}$ as:

$$
\min _{x} \frac{1}{2}(x-s)^{T}(x-s)+1^{T}|\Gamma x|
$$

where $\Gamma$ is the node-edge incidence matrix, $1^{T}|\Gamma x|$ measures change across edges in $\ell_{1}$ and $(x-s)^{T}(x-s)$ measures the deviation from the source $s$ in $\ell_{2}$. Problem 6 can be formulated as follows:

$$
\left.\begin{array}{ll}
\min _{x, t} & 1^{T} t+1 / 2(x-s)^{T}(x-s) \\
& t \geq A x \\
& t \geq-A x
\end{array}\right\} y^{+}
$$

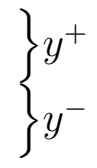

By introducing the Lagrangean variables $y^{+}, y^{-}$, we can write the dual as:

$$
\min _{x, t} \max _{y^{+} \geq 0, y^{-} \geq 0} 1^{T} t+1 / 2(x-s)^{T}(x-s)+y^{+^{T}}(-t-A x)+y^{-T}(-t+A x)
$$

Taking derivative with respect to $x$ yields

$$
x-s-A^{T}\left(y^{+}-y^{-}\right)=0
$$

Taking derivative with respect to $t$ yields

$$
y^{+}+y^{-}=1
$$

Let $y=y^{+}-y^{-}$. Since $y^{+}, y^{-} \geq 0$, we have $|y| \leq 1 \Longleftrightarrow y^{+}+y^{-}=1$ (to see this let $y^{+}=\frac{y+1}{2}$ and $y^{-}=\frac{-y+1}{2}$.) Plugging this back into the original formulation yields

$$
\xi(x)=\max _{|y| \leq 1}-\frac{1}{2} y^{T} \Gamma \Gamma^{T} y+y^{T} \Gamma s .
$$

It is well known that interior point methods can be applied to the above constrained problem by creating an unconstrained function $\hat{\xi}$ that replaces the linear 
constraint $|y| \leq 1$ with a log-barrier term ${ }^{2}, \lambda \log (1-y)+\lambda \log (1+y)$. The interior point optimization now amounts to Newton's method[6] on $\hat{\xi}(x)$. To uncover the computational complexity of the procedure we begin by calculating the gradient and hessian of $\hat{\xi}(x)$ and examine their structure:

$$
\begin{aligned}
\frac{\partial \hat{\xi}}{\partial y} & =-\Gamma \Gamma^{T} y+\Gamma s+\lambda \frac{1}{y-1}+\lambda \frac{1}{y+1} \\
\frac{\partial^{2} \hat{\xi}}{\partial y^{2}} & =-\Gamma \Gamma^{T}-\lambda \mathrm{d}(y-1)^{-2}-\lambda \mathrm{d}(y+1)^{-2}
\end{aligned}
$$

where $\mathrm{d}(x)$ promotes the vector $x$ to a diagonal matrix. Recall for Newton's method that the computational bottleneck is solution to linear system $Q^{t} y^{(t+1)}=$ $y^{t}$, where the hessian, $Q^{t}$ at iteration $t$ is given by eq. 9 .

Hence the algorithm is:

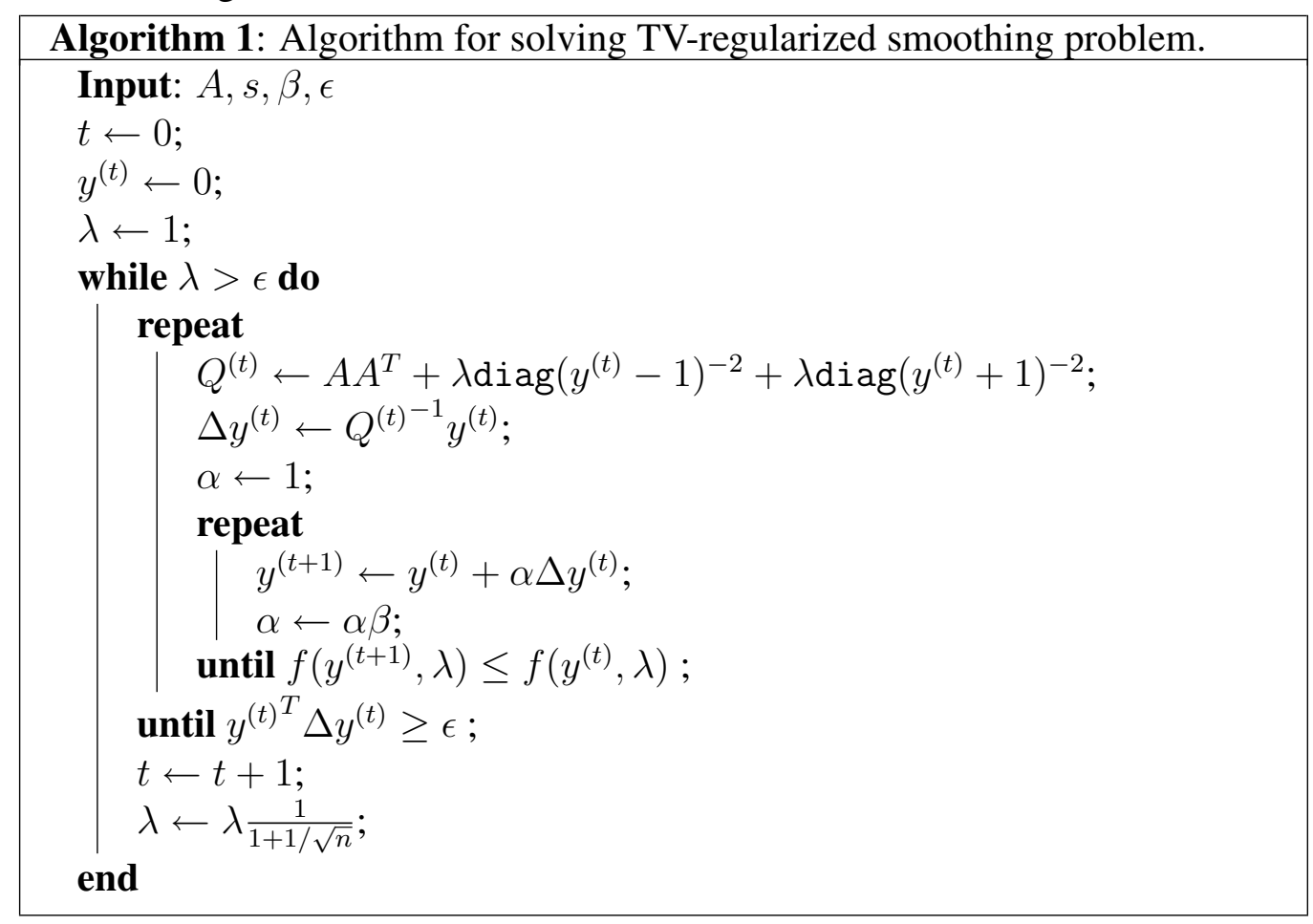

Observe that the hessian matrix is factored as $Q^{(t)}=\left(\Gamma \Gamma^{T}+C\right)$ where $C$ is a diagonal matrix. Using Binomial inverse theorem, we have $Q^{(t)}{ }^{-1}=C^{-1}-$

\footnotetext{
${ }^{2}$ Recall: As $\lambda \rightarrow 0$, we have $\lambda \log (x)=\left\{\begin{array}{ll}0, & x>0 \\ -\infty, & x \leq 0\end{array}\right.$, here we assume $\log (x)=-\infty$ if $x \leq 0$.
} 
$C^{-1}\left(I+\Gamma^{T} C^{-1} \Gamma\right)^{-1} C^{-1}$, where $I$ is the identity matrix. Since $C$ is a diagonal matrix, $C^{-1}$ is easy to compute. Further note that $\Gamma^{T} C^{-1} \Gamma$ is a weighted Laplacian matrix, so $I+\Gamma^{T} C^{-1} \Gamma$ is SDD. Given that Newton's method takes $O(\sqrt{n})$ many iterations [6], the total running time is at most $\tilde{O}\left(n^{3 / 2}\right)$ for the optimization of $\ell_{2}, \ell_{1}$.

\subsubsection{Non-Local Means}

Motivated by Efros' texture work [14], the non-local means (NLM) [9] energy functional has received a great deal of recent attention [8, 34, 35] due to its empirical performance in textured regions. We observe that the discrete instance of the functional can be written in terms of solving a weighted SDD. The NLM energy functional takes the following form:

$$
\min _{x}:\|x-s\|_{2}^{2}+\lambda \sum_{(i j) \in N}\left\|p_{i}-p_{j}\right\|_{W}^{2}
$$

where $s$ is the observed signal, $x$ the de-noised signal, $p_{i}$ is the image patch centered at location $i$, and $W$ and an empirical weighting function over the patch pairs $(i j)$.

The second term $\sum_{(i j) \in N}\left\|p_{i}-p_{j}\right\|_{W}^{2}$ in Eq 10 can be written out as a symmetric quadratic form. Where $R_{i}$ is the $k^{2}$ by $n$ restriction matrix, as in $\S 2.4$, that selects the pixels from patch $i$ and places them in a vector. The non-local term can now be written as

$$
\begin{aligned}
\sum_{(i j) \in N} w_{i j}\left(R_{i} x-R_{j} x\right)^{T}\left(R_{i} x-R_{j} x\right) & =x^{T}\left(\sum_{(i j) \in N} w_{i j}\left(R_{i}-R_{j}\right)^{T}\left(R_{i}-R_{j}\right)\right) x \\
& =x^{T} M x .
\end{aligned}
$$

The matrix $M$ is clearly positive semi-definite as it is the sum of products, further, we see that it is a weighted Laplacian matrix as $R_{i}-R_{j}$ terms are exactly edgenode incidence matrices over the patch pixels.

To solve the NLM problem replace the patch term $\lambda \sum_{(i j) \in N}\left\|p_{i}-p_{j}\right\|_{W}^{2}$, in eq 10, with $\lambda x^{T} M x$ and take the derivative with respect to $x$, setting to zero we arrive at linear system $(I+\lambda M) x=s$. The matrix $(I+\lambda M)$ is SDD as it the sum of SDD matrices, therefore $x$ can be found in $\tilde{O}(n)$ work. Note that the above is a single step due to the $\ell_{2}$ smoothness term, $\ell_{1}$ penalization of the patch smoothness is easily achieved by adapting the duality algorithm to NLM. 


\subsection{Clustering, Maps, Matting and Segmentation}

In recent years data clustering, embedding, image matting and image segmentation problems have been formulated as optimizations on combinatorial graphs representing the data $[40,32,2,48,11,30,18,12]$. Laplacians, normalized Laplacians and related linear operators of graphs arise naturally in formulating the objective functions and optimization procedures. In this section we briefly relate a handful of recent approaches that ultimately reduce to solving SDD linear systems.

As an example, the resistive network analogy (see §2.3) motivated an assisted segmentation method for images and volumes. Grady et al. [18, 41] exploited the relationship between graph Laplacians and random walks to segment images given sparse labels. This method requires a single solve and a sort to achieve it's solution (a cost is dominated the vertex sort in the planar case).

Similar work $[48,12,15]$ extends the NCuts and related objectives, discussed below, to include membership constraints yielding assisted clustering procedures. These approaches differ from the Random Walks procedure, and Linear Programming $^{3}$ based $k$-way min-cut approaches in that the relative sizes of the partitions are explicitly balanced.

\subsubsection{Eigencalculations in Vision}

Calculating a minimal, say $k$-dimensional, eigenspace of an SDD matrix forms the computational core of the spectral relaxation for NCuts [40], spectral clustering [32], Laplacian eigenmaps [2], diffusion maps [11], and the typical case for Levin et. al.'s image matting algorithm [30]. Recall that eigensystems satisfy the following equations for a Laplacian $A z_{i}=\phi_{i} z_{i}$ and generalize to $A x_{i}=\lambda_{i} D x_{i}$ where we assume $D$ is positive definite diagonal in general, and typically $D$ is the weighted degree matrix (see $\S 2.4$ ).

For the generalized problem, efficient computation of the $2 k$ quantities $\left(x_{i}, \lambda_{i}\right)$, such that $\lambda_{1} \leq \lambda_{i} \leq \lambda_{k+1}$, depends upon the relationship to a normalized Laplacian, $\mathcal{A}=D^{-1 / 2} A D^{-1 / 2}$. $\mathcal{A}$, possessed of the same eigenvalues $\left\{\lambda_{i}\right\}$ as the generalized problem, with eigenvectors $y_{i}$ that map to generalized vectors under the operator $D^{1 / 2}: x_{i}=D^{1 / 2} y_{i}$.

As $D$ is a positive operator $\mathcal{A}$ is SDD. We find the set of eigenvalues and vectors by inverse powering, i.e. repeated solves of the problem $\mathcal{A} q^{t+1}=q^{t}$,

\footnotetext{
${ }^{3}$ It is worth noting that the best theoretical upper bound for computing min-cut $\backslash$ max-flow can be obtained by solving SDD systems[42].
} 
coupled with a Krylov space method such as the Lanczos algorithm. In [43] the number of inverse powers required was shown to be $O(\log n)$ to calculate a vector with a Rayleigh quotient arbitrarily close to that of the minimal eigenspace. Thus the general case complexity of an eigencalculation remains $O\left(n \log ^{o(1)} n\right)$. In essence by employing the $\mathrm{CMG}$ solver we achieve approximate NCuts solutions in time roughly proportional to sorting the vertices (pixels) by value.

\section{Results}

Many computer vision problems naturally suggest a graph structure - for example the vertices often correspond to samples (e.g. pixels, patches, images), the edge set establishes pairwise comparisons or constraints encoded in the graph and the weights are either data driven (for clustering) or the result of an ongoing optimization procedure (weights in the $t^{t h}$ iteration of Newton's method).

In this section we demonstrate our general case combinatorial multigrid preconditioner (CMG) solver on a suite of applications taken from computer vision. The data is presented relative to the timing of a direct methods, found in MATLAB and LAPACK, for reference. We emphasize that our goal is not to perform numerical comparisons with any of the previous solvers, but rather to demonstrate that fast solvers with provable properties (such as CMG) are within the realm of practical implementation. The presented solver was written in a combination of $\mathrm{C}$ and MATLAB with modest attention paid to code optimization.

\subsubsection{Notation}

The weighted laplacian matrix $A$ can be factored as $A=\Gamma^{T} W \Gamma$ where $\Gamma$ is a edge-node incidence matrix, and $W$ is a nonnegative diagonal weight matrix over the edges. The normalized Laplacian is defined $\hat{A}=D^{-1 / 2} A D^{-1 / 2}=$ $I-D^{-1 / 2} G D^{-1 / 2}$ where $D$ is the weighted degree matrix and $G$ the weighted adjacency matrix. Recall that quadratic form $x^{T} A x$ can be written in several forms $x^{T} D x-x^{T} G x=\sum_{i} d_{i} x_{i}^{2}-2 \sum_{i j} w_{i j} x_{i} x_{j}=\sum_{i j} w_{i j}\left(x_{i}-x_{j}\right)^{2}$.

\subsection{SDD Linear Systems}

Sparse, unit weight, SDD linear systems arise in non-local means[9, 8], gradient inpainting [44, 31, 4], segmentation [17], regression and classification [49] and related data interpolation optimizations. For example, the in-painting functional 
$f(x)=\min _{x}:(\Gamma x-\Delta)^{T} W(\Gamma x-\Delta)$, where $\Delta$ is a vector of target gradient values to be exhibited in the image $x$ and $\Gamma$ is a generalized gradient operator, requires the solution to $\Gamma^{T} W \Gamma x=\Gamma^{T} W \Delta$. When $W$ is the identity and $\Gamma$ embodies a 4-connected topology these systems can be efficiently solved (provably) by geometric multigrid methods including the method described herein. For unit and weighted general planar systems the asymptotic complexity of solving $A x=b$ is $O(n)$ [29].

When $W$ is a more general nonnegative diagonal matrix and $\Gamma$ encodes nonplanar connectivity many multigrid methods will fail. Figure 3 shows such a class of problems in a log-log plot for weighted 3D graphs derived from a 3D CT Study of an oncology phantom - with the edge weighting: $w_{u v}=\exp \left(-\left(I_{u}-I_{v}\right)^{2} / \sigma^{2}\right)$ between neighboring voxels $u$ and $v$, where $I_{u}$ denotes the intensity of voxel $u$. Solving such 3D lattice SDDs, and general topologies, requires only $O\left(n \log ^{o(1)} n\right)$ [43] work, however we observe linear work empirically.

\subsubsection{Eigencalculations:}

Calculating a minimal, say $k$-dimensional, eigenspace of an SDD matrix forms the computational core of the spectral relaxation for NCuts [40], spectral clustering [32], Laplacian eigenmaps [2], diffusion maps [11], and the typical case for Levin et. al.'s image matting algorithm [30]. Recall that eigensystems satisfy the following equations for a Laplacian $A z_{i}=\phi_{i} z_{i}$ and generalize to $A x_{i}=\lambda_{i} D x_{i}$ where we assume $D$ is positive definite diagonal in general, and typically $D$ is the weighted degree matrix (see $\S 2.4$ ).

For the generalized problem, efficient computation of the $k$ eigenpairs $\left(x_{i}, \lambda_{i}\right)$, such that $\lambda_{1} \leq \lambda_{i} \leq \lambda_{k+1}$, depends upon the relationship to a normalized Laplacian, $\hat{A}$. $\hat{A}$ is possessed of the same eigenvalues $\left\{\lambda_{i}\right\}$ as the generalized problem, with eigenvectors $y_{i}$ that map to generalized vectors under the operator $D^{1 / 2}$ : $x_{i}=D^{1 / 2} y_{i}$.

We find the set of eigenvalues and vectors by inverse powering, i.e. repeated solves of the problem $\hat{A} q^{t+1}=q^{t}$, coupled with a Krylov space method such as the Lanczos algorithm. In [43] the number of inverse powers required was shown to be $O(\log n)$ to calculate a vector with a Rayleigh quotient arbitrarily close to that of the minimal eigenspace. Thus the general case complexity of an eigencalculation remains $O\left(n \log ^{o(1)} n\right)$. In essence by employing the CMG solver we achieve approximate NCuts solutions in time roughly proportional to sorting the vertices (pixels) by intensity. Timing results for estimating $\left(x_{2}, \lambda_{2}\right)$ on three panoramic landscape image derived graphs is shown in column 4 of Table 1 (edge 


\begin{tabular}{|l||r|r||r|r||r|r|}
\hline Size in Mega-pixels & “" & CMG & " $"+$ eigs & CMG+eigs & $\ell_{2}, \ell_{1}$ w “ $\backslash "$ & $\ell_{2}, \ell_{1}$ w CMG \\
\hline \hline $2 \mathrm{M}$ & $45 \mathrm{~s}$ & $\mathbf{8 s}$ & $1.6 \mathrm{~m}$ & $\mathbf{5 9 s}$ & $5.1 \mathrm{~m}$ & $\mathbf{3 1 s}$ \\
\hline $10 \mathrm{M}$ & $4.9 \mathrm{~m}$ & $\mathbf{2 2 s}$ & $7.6 \mathrm{~m}$ & $\mathbf{2 . 7 m}$ & $49.4 \mathrm{~m}$ & $\mathbf{3 . 7 m}$ \\
\hline $50 \mathrm{M}$ & $N A$ & $\mathbf{1 . 1 m}$ & $N A$ & $\mathbf{7 . 1 m}$ & $N A$ & $\mathbf{1 4 . 6 m}$ \\
\hline
\end{tabular}

Table 1: Two dimensional comparison with MATLAB for solutions on weighted 2D problems (with $O(|V|)$ super-lattice topology) at 2,10, and 50 megapixels. The construction of the CMG hierarchy takes less than 5 seconds for the $50 \mathrm{M}$ problem. For CMG, the majority of the compute time is consumed by the solver iteration, for direct methods computing the factorization is most time consuming.

weightings as in above).

\subsubsection{Convex Programming and Reweighted Problems:}

The optimization of convex functionals such as " $\ell_{2}-\ell_{1}$ Total Variation"[37], given by: $f(x)=\min _{x}:|| x-s \|_{2}+\lambda|\nabla x|_{1}$ for an input signal $s$, and related problems can be accomplished using Newton's method with log-barriers in $O\left(n^{1.5} \log ^{o(1)}(n)\right)$ time with modern solvers. For TV, the computational crux of each iteration is the solution of a SDD system with iteration dependent edge weights for the Laplacian modeling the $\ell_{1}$ penalty on spatial gradients $|\nabla x|_{1}$. As the program iterates, the weights on edges between regions of different intensity approach zero; such weightings radically violate the conditions required by most multi-grid methods. CMG is suitable for such weightings, and further, the clustering determined by the steps in $\S 3$ are generally preserved across iterations thus requiring linear work to update the preconditioner, unlike, direct methods which require recomputing the factorization from scratch. This property is also useful for other iterative methods such as robust least squares. Timing results on the panoramic image problems can be found in columns 5 and 6 of Table 1.

\section{Discussion}

Finally, by segregating the code for solvers (and eigen-calculations) from that of the applications we harvest improved modularity, reliability and factorizations of the system. Thus, 1) errors can be isolated to either the solver or the application, 2) as new solvers become available they can be easily adopted and perhaps most importantly 3) it relieves the application designer of the burden of implementing the state-of-the-art in solver technology. 


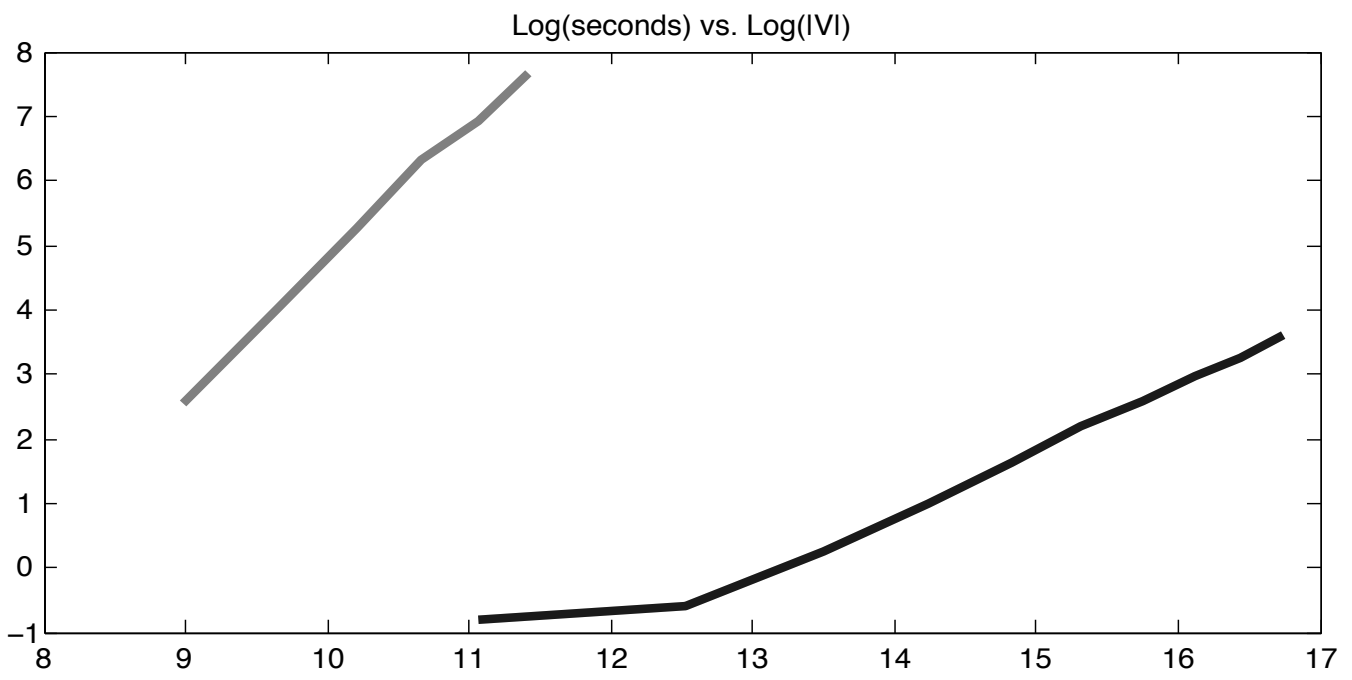

Figure 3: Relative speeds of the CMG solver (black) and MATLAB's " $\$ " operator (grey) on weighted graphs with 6 connected 3D lattices derived from a CT study. The $\mathrm{X}$-axis is shown in $\log$ scale over $|V|$, the number of variables in the system. For reference, the execution time for the $\mathrm{CMG}$ solver on a problem with 27 million variables is $\approx 50$ seconds.

We feel that significant improvements are still to be made in the solver and eigen-solver technology. We envision major new applications for these solvers in scientific computing, image processing, data-mining, and machine learning. Finally, to ease adoption of hybrid solvers, an implementation of the CMG solver will be made available in the near future.

\section{References}

[1] O. Axelsson. Iterative Solution Methods. Cambridge University Press, New York, NY, 1994. 4

[2] M. Belkin and P. Niyogi. Laplacian eigenmaps for dimensionality reduction and data representation. Neural Computation, 15(6):1373-1396, 2003. 2, 15, 17

[3] M. Bern, J. R. Gilbert, B. Hendrickson, N. Nguyen, and S. Toledo. Support-graph preconditioners. SIAM J. Matrix Anal. Appl., 4:930-951, 2006. 5

[4] P. Bhat, B. Curless, M. Cohen, and C. L. Zitnick. Fourier analysis of the $2 \mathrm{~d}$ screened poisson equation for gradient domain problems. In ECCV, 2008. 2, 11, 16 
[5] E. G. Boman and B. Hendrickson. Support theory for preconditioning. SIAM J. Matrix Anal. Appl., 25(3):694-717, 2003. 3, 5

[6] K. Boyd. Convex Optimization. Cambridge University Press, 1st edition, 2004. 13, 14

[7] A. Brandt. General highly accurate algebraic coarsening. Electronic Transactions on Numerical Analysis, 2000. 2

[8] T. Brox, O. Kleinschmidt, and D. Cremers. Efficient nonlocal means for denoising of textural patterns. Trans. on Image Processing, 2008. 2, 14, 16

[9] A. Buades, B. Coll, and J. Morel. Nonlocal image and movie denoising. IJCV, 76(2):123-139, 2008. 2, 14, 16

[10] T. Chan and J. Shen. Image Processing And Analysis: Variational, Pde, Wavelet, And Stochastic Methods. SIAM, 2005. 2, 11

[11] R. R. Coifman, S. Lafon, A. B. Lee, M. Maggioni, B. Nadler, F. Warner, and S. W. Zucker. Diffusion maps geometric diffusions as a tool for harmonic analysis and structure definition of data. PNAS, 102(21):7426-7431, May 2005. 2, 15, 17

[12] T. Cour and J. Shi. Solving markov random fields with spectral relaxation. AISTATS, 2007. 2, 15

[13] P. G. Doyle and J. L. Snell. Random walks and electric networks, 2000. 4

[14] A. A. Efros and T. K. Leung. Texture synthesis by non-parametric sampling. ICCV, 2:1033-1038, 1999. 14

[15] A. P. Eriksson, C. Olsson, and F. Kahl. Normalized cuts revisited: A reformulation for segmentation with linear grouping constraints. In ICCV, pages 1-8. IEEE, 2007. 15

[16] G. Gilboa and S. Osher. Nonlocal operators with applications to image processing. Multiscale Modeling and Simulation, July 2007. 2

[17] L. Grady. Multilabel random walker image segmentation using prior models. In $C V P R$, volume 1 of $C V P R$, pages 763-770, San Diego, June 2005. IEEE, IEEE. 16

[18] L. Grady. Random walks for image segmentation. IEEE Trans. on Pattern Analysis and Machine Intelligence, 2(11):1768-1783, 2006. 2, 15

[19] L. Grady. A lattice-preserving multigrid method for solving the inhomogeneous poisson equations used in image analysis. ECCV, 5303:252-264, 2008. 2, 7, 8

[20] L. Grady and C. Alvino. Reformulating and optimizing the mumford-shah functional on a graph - a faster, lower energy solution. ECCV, 5302:248-261, 2008. 2, 10

[21] L. Grady and A. K. Sinop. Fast approximate random walker segmentation using eigenvector precomputation. In CVPR. IEEE Computer Society, IEEE, June 2008. 2 
[22] K. Gremban. Combinatorial Preconditioners for Sparse, Symmetric, Diagonally Dominant Linear Systems. PhD thesis, Carnegie Mellon University, Pittsburgh, October 1996. CMU CS Tech Report CMU-CS-96-123. 3, 5, 6

[23] B. Horn. Shape from shading: A method for obtaining the shape of a smooth opaque object from one view. Technical Report 232, MIT AI Laboratory, November 1970. 2

[24] B. Horn. Determining lightness from an image. Computer Graphics and Image Processing, 3(1):277-299, 1974. 2

[25] B. Horn. Determining optical flow. MIT AI Laboratory, 17(1):185-203, 1981. 2

[26] A. Joshi. Topics in Optimization and Sparse Linear Systems. PhD thesis, University of Illinois at Urbana Champaing, 1997. 3

[27] I. Koutis. Combinatorial and algebraic algorithms for optimal multilevel algorithms. PhD thesis, Carnegie Mellon University, Pittsburgh, May 2007. CMU CS Tech Report CMU-CS-07-131. 3

[28] I. Koutis and G. L. Miller. "a linear work, $o\left(n^{1 / 6}\right)$ time, parallel algorithm for solving planar laplacians". In Proc. 18th ACM-SIAM Symposium on Discrete Algorithms (SODA 2007), 2007. 3

[29] I. Koutis and G. L. Miller. Graph partitioning into isolated, high conductance clusters: Theory, computation and applications to preconditioning. In Symposiun on Parallel Algorithms and Architectures (SPAA), 2008. 5, 6, 7, 8, 10, 17

[30] A. Levin, A. Rav-Acha, and D. Lischinski. Spectral matting. In $C V P R$, 2007. 2, 15, 17

[31] J. McCann and N. S. Pollard. Real-time gradient-domain painting. SIGGRAPH, 27(3), 2008. 2, 11, 16

[32] A. Ng, M. Jordan, and Y. Weiss. On spectral clustering: Analysis and an algorithm. In NIPS, 2002. 2, 15, 17

[33] P. Perona and J. Malik. Scale-space and edge detection using anisotropic diffusion. PAMI, 7(12):629-639, 1990. 2

[34] G. Peyre, S. Bougleux, and L. Cohen. Non-local regularization of inverse problems. In ECCV, volume 5304, pages 57-68, 2008. 11, 14

[35] M. Protter, M. Elad, H. Takeda, and P. Milanfar. Generalizing the nonlocal-means to super-resolution reconstruction. Trans. on Image Processing, 18(1):36-51, 2009. 11,14

[36] D. L. Ruderman and W. Bialek. Statistics of natural images: scaling in the woods. NIPS, 1994. 12

[37] L. Rudin, S. Osher, and E. Fatemi. Nonlinear total variation based noise removal algorithm. Physica D, 1(60):259-268, 1992. 2, 11, 12, 18 
[38] J. W. Ruge and K. Stüben. Algebraic multigrid (AMG). In S. F. McCormick, editor, Multigrid Methods, volume 3 of Frontiers in Applied Mathematics, pages 73-130. SIAM, Philadelphia, PA, 1987. 2

[39] E. Sharon, A. Brandt, and R. Basri. Segmentation and boundary detection using multiscale intensity measurements. In CVPR. IEEE Computer Society, IEEE, 2001. 2

[40] J. Shi and J. Malik. Normalized cuts and image segmentation. In PAMI, 2000. 2, 15,17

[41] A. K. Sinop and L. Grady. A seeded image segmentation framework unifying graph cuts and random walker which yields a new algorithm. In ICCV. IEEE Computer Society, IEEE, Oct. 2007. 15

[42] D. A. Spielman and S. I. Daitch. Faster approximate lossy generalized flow via interior point algorithms. In Proceedings of the 40th Annual ACM Symposium on Theory of Computing, May 2008. 15

[43] D. A. Spielman and S.-H. Teng. Nearly-linear time algorithms for preconditioning and solving symmetric, diagonally dominant linear systems, 2006. 3, 16, 17

[44] R. Szeliski. Locally adapted hierarchical basis preconditioning. SIGGRAPH, 25(3):1135-1143, August 2006. 2, 11, 16

[45] U. Trottenberg, A. Schuller, and C. Oosterlee. Multigrid. Academic Press, 1st edition, 2000. 9

[46] P. M. Vaidya. Solving linear equations with symmetric diagonally dominant matrices by constructing good preconditioners. A talk based on this manuscript, October 1991. 3

[47] A. Witkin. Scale-space filtering. IJCAI, pages 1019-1022, August 1983. 2

[48] S. Yu and J. Shi. Segmentation given partial grouping constraints. PAMI, 26(2):173183, 2004. 2, 15

[49] X. Zhu, Z. Ghahramani, and J. D. Lafferty. Semi-supervised learning using gaussian fields and harmonic functions. ICML, 2003. 16 